PROCEEDINGS OF THE

AMERICAN MATHEMATICAL SOCIETY

Volume 133, Number 6, Pages 1865-1872

S 0002-9939(04)07766-

Article electronically published on December 20, 2004

\title{
ON THE KERNEL OF THE MAGNUS REPRESENTATION OF THE TORELLI GROUP
}

\author{
MASAAKI SUZUKI \\ (Communicated by Ronald A. Fintushel)
}

\begin{abstract}
From our previous paper, it is known that the Magnus representation of the Torelli group is not faithful. In this paper, we characterize the kernel of its representation for a certain kind of elements.
\end{abstract}

\section{INTRODUCTION}

The linearity of the mapping class group of a surface of genus $g \geq 2$ has been one of the well-known open problems. A group is called linear if it admits a finitedimensional faithful representation. Recently, Korkmaz $[\mathrm{K}]$, Bigelow and Budney B-B proved that the mapping class group of a closed surface of genus 2 is linear. However, it still remains open for higher genera. Then it is significant to discuss whether some representations of the mapping class groups are faithful and to determine the kernel.

Let $\Sigma_{g, 1}$ be an oriented surface obtained from a closed surface of genus $g$ by removing an open disk. We denote by $\mathcal{M}_{g, 1}$ the mapping class group of $\Sigma_{g, 1}$ relative to the boundary, that is, the group of path components of the group of orientation preserving diffeomorphisms of $\Sigma_{g, 1}$ which restrict to the identity on the boundary. Let $\mathcal{I}_{g, 1}$ be the Torelli group of $\Sigma_{g, 1}$, namely the normal subgroup of $\mathcal{M}_{g, 1}$ consisting of all the elements which act trivially on the first homology group of $\Sigma_{g, 1}$.

The Magnus representations of various subgroups of the automorphism group of a free group are defined by making use of the Fox derivation [F]; see [Bir] for details. The Magnus representation for the Torelli group

$$
r_{1}: \mathcal{I}_{g, 1} \rightarrow G L(2 g ; \mathbb{Z}[H])
$$

was introduced in [M1, where $H=H_{1}\left(\Sigma_{g, 1} ; \mathbb{Z}\right)$. From our previous paper [S1, the representation $r_{1}$ is not faithful for $g \geq 2$. Thus it makes sense to study the kernel of $r_{1}$. In this paper, we characterize the kernel of $r_{1}$ for the commutator of two BSCC maps, where the Dehn twist along a bounding simple closed curve is called a BSCC map. The following is one of the main results of this paper.

Received by the editors October 16, 2003 and, in revised form, February 23, 2004.

2000 Mathematics Subject Classification. Primary 57M05; Secondary 20F34, 57N05.

Key words and phrases. Magnus representation, mapping class group, faithful.

The author was supported in part by JSPS Research Fellowships for Young Scientists.

(C)2004 American Mathematical Society

Reverts to public domain 28 years from publication 


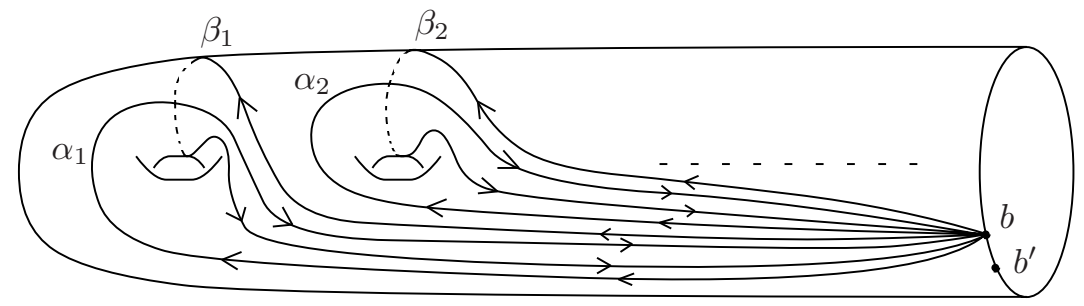

FiguRE 1. Generators of $\Gamma_{0}$ and base points $b, b^{\prime}$

Corollary 4.4 The commutator of two BSCC maps $\varphi_{1}, \varphi_{2}$ belongs to the kernel of $r_{1}$ if and only if the characteristic polynomial of the Magnus matrix of the product $\varphi_{1} \varphi_{2}$ is trivial. Here the Magnus matrix means the image of $r_{1}$ for a mapping class.

In Section 2, we will recall the definitions of the Magnus representation of the mapping class group and the Torelli group.

In Section 3, we will give a certain pairing for two curves on $\Sigma_{g, 1}$ and show the relationship with the pairing and the kernel of $r_{1}$.

In Section 4 we will introduce another pairing for two curves on $\Sigma_{g, 1}$ in order to obtain additional information of the kernel of $r_{1}$.

\section{Definition of the Magnus representation of the Torelli group}

In this section, we recall the definitions of the Magnus representation for the mapping class group and the Torelli group from [M1, [S1] and [S4].

Let $\mathbb{Z}\left[\Gamma_{0}\right]$ be the integral group ring of $\Gamma_{0}=\pi_{1}\left(\Sigma_{g, 1}, b\right)$. We fix a system of generators $\alpha_{1}, \ldots, \alpha_{g}, \beta_{1}, \ldots, \beta_{g}$ of the free group $\Gamma_{0}$ as shown in Figure 1 . Let us simply write $\gamma_{1}, \ldots, \gamma_{2 g}$ for them.

Definition 2.1. We call the mapping

$$
\begin{aligned}
r: \mathcal{M}_{g, 1} & \longrightarrow G L\left(2 g ; \mathbb{Z}\left[\Gamma_{0}\right]\right) \\
\varphi & \longmapsto\left(\frac{\overline{\partial \varphi\left(\gamma_{j}\right)}}{\partial \gamma_{i}}\right)_{i, j}
\end{aligned}
$$

the Magnus representation for the mapping class group, where $\frac{\partial}{\partial \gamma_{i}}: \mathbb{Z}\left[\Gamma_{0}\right] \rightarrow \mathbb{Z}\left[\Gamma_{0}\right]$ is the Fox derivation and ${ }^{-}: \mathbb{Z}\left[\Gamma_{0}\right] \rightarrow \mathbb{Z}\left[\Gamma_{0}\right]$ is the antiautomorphism induced by the mapping $\gamma \mapsto \gamma^{-1}$.

This mapping is not a homomorphism but a crossed homomorphism.

Proposition 2.2 (Morita [M1]). For any two elements $\varphi, \psi \in \mathcal{M}_{g, 1}$, we have

$$
r(\varphi \psi)=r(\varphi) \cdot{ }^{\varphi} r(\psi)
$$

where $\varphi_{r}(\psi)$ denotes the matrix obtained from $r(\psi)$ by applying the automorphism $\varphi: \mathbb{Z}\left[\Gamma_{0}\right] \rightarrow \mathbb{Z}\left[\Gamma_{0}\right]$ on each entry.

It follows that if this mapping $r$ is restricted to the Torelli group $\mathcal{I}_{g, 1}$ and if the coefficients are reduced to $\mathbb{Z}[H]$, then we obtain the following genuine representation:

$$
r_{1}: \mathcal{I}_{g, 1} \longrightarrow G L(2 g ; \mathbb{Z}[H]) .
$$


Here the reduction is induced by the abelianization $\mathfrak{a}: \Gamma_{0} \rightarrow H$, and $r_{1}$ denotes the composition $r^{\mathfrak{a}}$ of the mapping $r$ by the abelianization $\mathfrak{a}$. We call $r_{1}$ the Magnus representation of the Torelli group.

We have another definition of this representation (see [S4]). Let $p: \widehat{\Sigma} \rightarrow \Sigma_{g, 1}$ be the universal abelian covering, that is, the regular covering corresponding to the abelianization. An arbitrary element of the Torelli group induces an automorphism of $H_{1}\left(\widehat{\Sigma}, p^{-1}(b) ; \mathbb{Z}\right)$ as a free $\mathbb{Z}[H]$-module of rank $2 g$. Therefore we get the following representation:

$$
r_{1}: \mathcal{I}_{g, 1} \longrightarrow G L(2 g ; \mathbb{Z}[H])
$$

\section{A HigheR INTERSECTION NUMBER OF TWO LOOPS AND THE KERNEL OF $r_{1}$}

The non-triviality of the kernel of $r_{1}$ for $g \geq 2$ is proved in S1. Moreover, it is proved in $\mathrm{S2}$ that none of the terms of the lower central series of $\mathcal{I}_{g, 1}$ is contained in the kernel. Then it is interesting to characterize and determine the kernel.

First, we define a pairing of two loops on $\Sigma_{g, 1}$. This pairing is useful to give information about the kernel of $r_{1}$. Choose base points $b$ and $b^{\prime}$ on $\partial \Sigma_{g, 1}$ as depicted in Figure 1, Fix a point $\hat{b}$, which is a lift of $b$ to the universal abelian covering $\widehat{\Sigma}$. The point $\hat{b}^{\prime}$, which is a lift of $b^{\prime}$, is determined as follows. We denote by $b b^{\prime}$ the path on $\partial \Sigma_{g, 1}$ from $b$ to $b^{\prime}$ with an orientation opposite to that of $\Sigma_{g, 1}$. Let $\widehat{b b^{\prime}}$ be the lift of $b b^{\prime}$ to $\widehat{\Sigma}$ starting at $\hat{b}$. Then we set $\hat{b}^{\prime}$ for the endpoint of $\hat{b b^{\prime}}$.

Definition 3.1. Let $c_{1}, c_{2}$ be two oriented loops on $\Sigma_{g, 1}$ based at $b, b^{\prime}$ respectively. We define

$$
\left\langle c_{1}, c_{2}\right\rangle_{H}=\sum_{h \in H}\left(h \hat{c}_{1}, \hat{c}_{2}\right) h .
$$

Here $\hat{c}_{1}$ is the lift of $c_{1}$ to $\widehat{\Sigma}$ starting at $\hat{b}, \hat{c}_{2}$ is the lift of $c_{2}$ to $\widehat{\Sigma}$ starting at $\hat{b}^{\prime}$ and $(\cdot, \cdot)$ denotes the algebraic intersection number of two arcs. We write $h \hat{c}_{1}$ for the arc obtained by an element $h$ of the covering transformation group $H$ acting on $\hat{c}_{1}$.

Suppose that $c_{1}$ and $c_{2}$ are bounding simple closed curves on $\Sigma_{g, 1}$, where bounding means 0 -homologous. If we regard $c_{1}, c_{2}$ as oriented loops based at $b, b^{\prime}$ respectively, then we can compute the pairing $\left\langle c_{1}, c_{2}\right\rangle_{H}$ up to multiplication by \pm 1 and by an element of $H$. That is to say, the pairing $\left\langle c_{1}, c_{2}\right\rangle_{H}$ depends on how $c_{1}, c_{2}$ are represented as loops. However, whether $\left\langle c_{1}, c_{2}\right\rangle_{H}$ is zero or not does not depend on the choices, and we will use this fact.

Proposition 3.2. Suppose that $c_{1}$ and $c_{2}$ are two bounding simple closed curves on $\Sigma_{g, 1}$, and $\varphi_{1}$ and $\varphi_{2}$ the Dehn twists along $c_{1}$ and $c_{2}$ respectively. If $\left\langle c_{1}, c_{2}\right\rangle_{H}=0$, then $\left[\varphi_{1}, \varphi_{2}\right] \in \operatorname{ker} r_{1}$.

Proof. We denote by $\widehat{\varphi}_{*}$ the automorphism of $H_{1}\left(\widehat{\Sigma}, p^{-1}(b) ; \mathbb{Z}\right)$ induced by a diffeomorphism $\varphi$ of $\Sigma_{g, 1}$ representing an element of $\mathcal{M}_{g, 1}$. Let $\hat{c}_{1}, \hat{c}_{2}$ be lifts of $c_{1}, c_{2}$ to $\widehat{\Sigma}$ respectively. Then $\left[\hat{c}_{1}\right],\left[\hat{c}_{2}\right]$ belong to $H_{1}\left(\widehat{\Sigma}, p^{-1}(b) ; \mathbb{Z}\right)$. Since $\left\langle c_{1}, c_{2}\right\rangle_{H}=0$, the intersection number $\left(\hat{c}_{1}, \hat{c}_{2}\right)$ equals zero. For a loop $c$ based at $b$, we denote by $\hat{c}$ a lift of $c$ to $\widehat{\Sigma}$. Then we have an element $[\hat{c}]$ of $H_{1}\left(\widehat{\Sigma}, p^{-1}(b) ; \mathbb{Z}\right)$ and

$$
\widehat{\varphi}_{i_{*}}([\hat{c}])=[\hat{c}]+\left(\hat{c}_{i}, \hat{c}\right)\left[\hat{c}_{i}\right], \quad i=1,2 .
$$




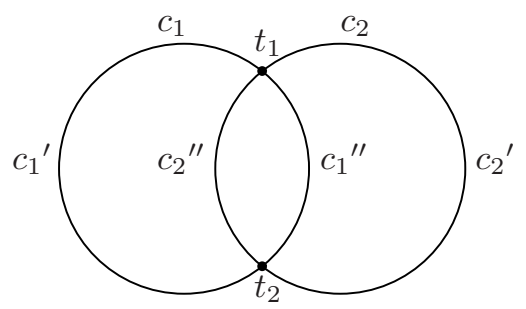

FIGURE 2. Geometric intersection number 2

Then we obtain

$$
\begin{aligned}
{\widehat{\varphi_{1}}}_{*} \circ{\widehat{\varphi_{2}}}_{*}([\hat{c}]) & =\widehat{\varphi}_{1 *}\left([\hat{c}]+\left(\hat{c}_{2}, \hat{c}\right)\left[\hat{c}_{2}\right]\right) \\
& =[\hat{c}]+\left(\hat{c}_{1}, \hat{c}\right)\left[\hat{c}_{1}\right]+\left(\hat{c}_{2}, \hat{c}\right)\left[\hat{c}_{2}\right] \\
& =\widehat{\varphi}_{2} \circ \widehat{\varphi}_{1 *}([\hat{c}]) .
\end{aligned}
$$

It follows that $\widehat{\varphi_{1}}$ commutes with $\widehat{\varphi_{2}}$, and this completes the proof.

Corollary 3.3. Suppose that $c_{1}$ and $c_{2}$ are two bounding simple closed curves. If the geometric intersection number of $c_{1}$ and $c_{2}$ is two, then $\left[\varphi_{1}, \varphi_{2}\right] \in \operatorname{ker} r_{1}$.

Proof. Let $t_{1}, t_{2}$ be the intersection points. Also, let $c_{i}{ }^{\prime}$ be the subarcs of $c_{i}$ from $t_{1}$ to $t_{2}$, and the subarcs $c_{i}{ }^{\prime \prime}$ from $t_{2}$ to $t_{1}$; see Figure 2. The number of the terms of $\left\langle c_{1}, c_{2}\right\rangle_{H}$ is two. Each value of the terms is decided by the value at $t_{1}$ and $t_{2}$ respectively. We consider loops $c_{1}{ }^{\prime} c_{2}{ }^{\prime \prime}, c_{1}{ }^{\prime} c_{2}{ }^{-1}, c_{2}{ }^{\prime} c_{1}{ }^{\prime \prime}$ and $c_{2}{ }^{\prime \prime} c_{1}{ }^{\prime \prime-1}$, where $c^{-1}$ is the same arc as $c$ with the opposite orientation. All of these are bounding simple closed curves. It follows that the value at $t_{1}$ is -1 times that of $t_{2}$. Then $\left\langle c_{1}, c_{2}\right\rangle_{H}=0$. By Proposition 3.2, this completes the proof.

\section{Another pairing of Bounding Simple Closed CuRVeS AND THE KERNEL OF $r_{1}$}

We define another pairing for two bounding simple closed curves:

$$
\left\langle\left\langle c_{1}, c_{2}\right\rangle\right\rangle=-\left\langle c_{1}, c_{2}\right\rangle_{H} \cdot\left\langle c_{2}, c_{1}\right\rangle_{H} .
$$

The pairing $\langle\cdot, \cdot\rangle_{H}$ depends on the manner of assigning orientations and attaching basepoints to two bounding simple closed curves. However, the way in which this is done does not have an effect on the pairing $\langle\langle\cdot, \cdot\rangle\rangle$. That is, we obtain the following lemma.

Lemma 4.1. Let $c_{1}, c_{2}$ be two bounding simple closed curves on $\Sigma_{g, 1}$. Then we have

(1) $\left\langle\left\langle c_{1}, c_{2}\right\rangle\right\rangle=\left\langle\left\langle c_{2}, c_{1}\right\rangle\right\rangle$

(2) $\left\langle\left\langle\gamma c_{1} \gamma^{-1}, c_{2}\right\rangle\right\rangle=\left\langle\left\langle c_{1}, c_{2}\right\rangle\right\rangle$,

(3) $\left\langle\left\langle c_{1}^{-1}, c_{2}\right\rangle\right\rangle=\left\langle\left\langle c_{1}, c_{2}\right\rangle\right\rangle$

where $\gamma$ is a loop based at $b$ and $c_{1}^{-1}$ is the same loop as $c_{1}$ with the opposite orientation. 
We recall the following before proving Lemma 4.1

Theorem 4.2 (Morita [M1]). There exists a matrix $\widetilde{J}$ such that for any element $f \in \mathcal{M}_{g, 1}$ the following equality holds:

$$
\overline{{ }^{t} r(f)} \widetilde{J} r(f)={ }^{f} \widetilde{J} .
$$

This means that the Magnus representation of the mapping class group is symplectic in a sense. The explicit expression for $\widetilde{J}$ can be found in [M1] and [S4] and is not included in this paper.

In this section, $\vec{c}$ denotes ${ }^{t}\left(\mathfrak{a}\left(\frac{\partial c}{\partial \gamma_{1}}\right), \ldots, \mathfrak{a}\left(\frac{\partial c}{\partial \gamma_{2 g}}\right)\right)$.

Proof of Lemma 4.1. (1) This is obvious from the definition of the pairing $\langle\langle\cdot, \cdot\rangle\rangle$.

(2) We can consider $\gamma$ as an element of $\Gamma_{0}$ naturally. Because

$$
\begin{aligned}
\mathfrak{a}\left(\frac{\partial \gamma c_{1} \gamma^{-1}}{\partial \gamma_{i}}\right) & =\mathfrak{a}\left(\frac{\partial \gamma}{\partial \gamma_{i}}\right)+\mathfrak{a}(\gamma) \mathfrak{a}\left(\frac{\partial c_{1}}{\partial \gamma_{i}}\right)+\mathfrak{a}(\gamma) \mathfrak{a}\left(c_{1}\right) \mathfrak{a}\left(\frac{\partial \gamma^{-1}}{\partial \gamma_{i}}\right) \\
& =\mathfrak{a}(\gamma) \mathfrak{a}\left(\frac{\partial c_{1}}{\partial \gamma_{i}}\right),
\end{aligned}
$$

then we get

$$
\overrightarrow{\gamma c_{1} \gamma^{-1}}=\mathfrak{a}(\gamma) \overrightarrow{c_{1}} .
$$

By [S4 Lemma 4.4], we have $\left\langle c_{1}, c_{2}\right\rangle_{H}=-{ }^{t} \overrightarrow{c_{2}} J_{1} \overrightarrow{\overline{c_{1}}}$, where $\mathfrak{a}(\widetilde{J})=J_{1}$. Therefore

$$
\begin{aligned}
\left\langle\left\langle\gamma c_{1} \gamma^{-1}, c_{2}\right\rangle\right\rangle & =-{ }^{t} \overrightarrow{c_{2}} J_{1} \overline{\mathfrak{a}(\gamma)} \overline{\overline{c_{1}}} t \mathfrak{a}(\gamma) \overrightarrow{c_{1}} J_{1} \overline{\overrightarrow{c_{2}}} \\
& =-{ }^{t} \overrightarrow{c_{2}} J_{1} \overline{\overline{c_{1}}} t \overrightarrow{c_{1}} J_{1} \overline{\overrightarrow{c_{2}}} \\
& =\left\langle\left\langle c_{1}, c_{2}\right\rangle\right\rangle .
\end{aligned}
$$

(3) Since

$$
\mathfrak{a}\left(\frac{\partial c_{1}^{-1}}{\partial \gamma_{i}}\right)=\mathfrak{a}\left(c_{1}^{-1}\right) \mathfrak{a}\left(\frac{\partial c_{1}}{\partial \gamma_{i}}\right)=-\mathfrak{a}\left(\frac{\partial c_{1}}{\partial \gamma_{i}}\right),
$$

we deduce this lemma.

The relation between the pairing $\langle\langle\cdot, \cdot\rangle\rangle$ and the Magnus representation $r_{1}$ of the Torelli group can be expressed as the following formula.

Theorem 4.3. Suppose that $c_{1}$ and $c_{2}$ are two bounding simple closed curves on $\Sigma_{g, 1}$. Then we obtain

$$
\left\langle\left\langle c_{1}, c_{2}\right\rangle\right\rangle=\operatorname{tr}\left(I_{2 g}-r_{1}\left(\varphi_{1} \varphi_{2}\right)\right)=2 g-\operatorname{tr}\left(r_{1}\left(\varphi_{1} \varphi_{2}\right)\right)
$$

where $\varphi_{1}, \varphi_{2}$ are the Dehn twists along $c_{1}, c_{2}$ respectively.

Proof. Any bounding simple closed curve can be written as $f\left(d_{k}\right)$ for a certain element $f \in \mathcal{M}_{g, 1}$ and for a bounding simple closed curve $d_{k}$, which is shown in Figure 3. First, we will prove the statement in the case $c_{1}=f\left(d_{i}\right), c_{2}=d_{j}$. That is, we will consider the case $\varphi_{1}=f \psi_{i} f^{-1}, \varphi_{2}=\psi_{j}$, where $\psi_{k}$ is the Dehn twist along $d_{k}$. By Lemma 4.1, we can assume that $c_{1}$ and $c_{2}$ have expressions such as

$$
c_{1}=f\left(\left[\beta_{i}, \alpha_{i}\right] \cdots\left[\beta_{1}, \alpha_{1}\right]\right), \quad c_{2}=\left[\beta_{j}, \alpha_{j}\right] \cdots\left[\beta_{1}, \alpha_{1}\right] .
$$

We see from [S3] that

$$
r_{1}\left(\psi_{k}\right)=I_{2 g}+a_{k} b_{k}
$$




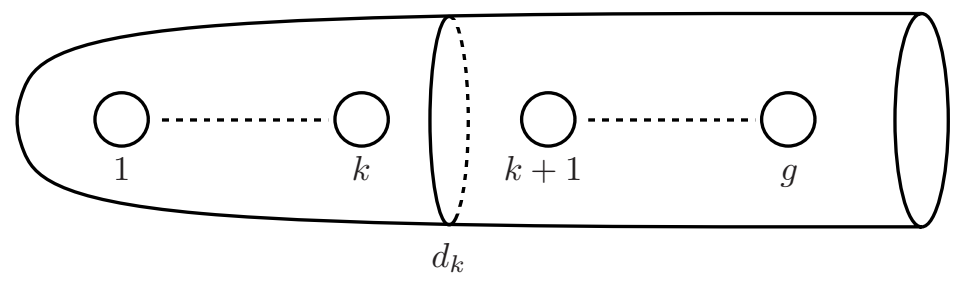

FiguRE 3. Bounding simple closed curve

Here

$$
\begin{aligned}
& a_{k}={ }^{t}(\bar{y}_{1}-1 \cdots \bar{y}_{k}-1 \underbrace{0 \cdots 0} \underbrace{0}_{g-k \text { times }} 1-\bar{x}_{1} \cdots 1-\bar{x}_{k} \underbrace{0 \cdots 0}_{g-k \text { times }}), \\
& b_{k}=(1-\bar{x}_{1} \cdots 1-\bar{x}_{k} \underbrace{0 \cdots 0}_{g-k \text { times }} 1-\bar{y}_{1} \cdots 1-\bar{y}_{k} \underbrace{0 \cdots 0}_{g-k}{ }_{\text {times }}^{0 \cdots},
\end{aligned}
$$

and $x_{i}, y_{i}$ are the homology classes of $\alpha_{i}, \beta_{i}$ respectively. Note that $\operatorname{tr}\left(a_{k} b_{k}\right)=$ $b_{k} a_{k}=0$. We denote by $r^{\mathfrak{a}}$ the composition of the mapping $r$ by the abelianization $\mathfrak{a}: \mathbb{Z}\left[\Gamma_{0}\right] \rightarrow \mathbb{Z}[H]$. If we consider elements of the Torelli group, we write $r_{1}$ for $r^{\mathfrak{a}}$ as before. By the abelianization, Theorem 4.2 can be stated as

$$
\overline{{ }^{t} r^{\mathfrak{a}}(f)} J_{1} r^{\mathfrak{a}}(f)={ }^{f} J_{1} .
$$

The following equalities can be checked easily:

$$
b_{k} J_{1}{ }^{-1}=\overline{{ }^{t} a_{k}}, \quad \overline{{ }^{t} a_{k}} J_{1}=b_{k} .
$$

We will compute $\overline{\overline{c_{1}}}$ by an explicit calculation. Since

$$
\begin{aligned}
\mathfrak{a}\left(\frac{\partial c_{1}}{\partial \gamma_{l}}\right) & \sum_{k=1}^{i} \mathfrak{a}\left(\frac{\partial f\left(\left[\beta_{k}, \alpha_{k}\right]\right)}{\partial \gamma_{l}}\right) \\
= & \sum_{k=1}^{i}\left\{\mathfrak{a}\left(\frac{\partial f\left(\beta_{k}\right)}{\partial \gamma_{l}}\right)+\mathfrak{a}\left(f\left(\beta_{k}\right)\right) \cdot \mathfrak{a}\left(\frac{\partial f\left(\alpha_{k}\right)}{\partial \gamma_{l}}\right)\right. \\
& \left.\quad+\mathfrak{a}\left(f\left(\beta_{k}\right)\right) \cdot \mathfrak{a}\left(f\left(\alpha_{k}\right)\right) \cdot \mathfrak{a}\left(\frac{\partial f\left(\beta_{k}^{-1}\right)}{\partial \gamma_{l}}\right)+\mathfrak{a}\left(f\left(\alpha_{k}\right)\right) \cdot \mathfrak{a}\left(\frac{\partial f\left(\alpha_{k}{ }^{-1}\right)}{\partial \gamma_{l}}\right)\right\} \\
= & \sum_{k=1}^{i}\left\{\mathfrak{a}\left(\frac{\partial f\left(\beta_{k}\right)}{\partial \gamma_{l}}\right)+f\left(y_{k}\right) \cdot \mathfrak{a}\left(\frac{\partial f\left(\alpha_{k}\right)}{\partial \gamma_{l}}\right)\right. \\
= & \left.\quad-f\left(x_{k}\right) \cdot \mathfrak{a}\left(\frac{\partial f\left(\beta_{k}\right)}{\partial \gamma_{l}}\right)-\mathfrak{a}\left(\frac{\partial f\left(\alpha_{k}\right)}{\partial \gamma_{l}}\right)\right\} \\
= & \sum_{k=1}^{i}\left\{\left(f\left(y_{k}\right)-1\right) \cdot \mathfrak{a}\left(\frac{\partial f\left(\alpha_{k}\right)}{\partial \gamma_{l}}\right)+\left(1-f\left(x_{k}\right)\right) \cdot \mathfrak{a}\left(\frac{\partial f\left(\beta_{k}\right)}{\partial \gamma_{l}}\right)\right\},
\end{aligned}
$$

we obtain

$$
\overline{\overline{c_{1}}}=r^{\mathfrak{a}}(f) \cdot{ }^{f} a_{i}
$$


Similarly, $\overrightarrow{c_{2}}=\overline{a_{j}}$. Therefore,

$$
\begin{aligned}
\operatorname{tr} & \left(I_{2 g}-r_{1}\left(\varphi_{1} \varphi_{2}\right)\right) \\
& =\operatorname{tr}\left(I_{2 g}-r^{\mathfrak{a}}(f) \cdot{ }^{f} r_{1}\left(\psi_{i}\right) \cdot r^{\mathfrak{a}}(f)^{-1} \cdot r_{1}\left(\psi_{j}\right)\right) \\
& =\operatorname{tr}\left(I_{2 g}-r^{\mathfrak{a}}(f) \cdot\left(I_{2 g}+{ }^{f} a_{i}{ }^{f} b_{i}\right) \cdot r^{\mathfrak{a}}(f)^{-1} \cdot\left(I_{2 g}+a_{j} b_{j}\right)\right) \quad \text { by (4.1) } \\
& =\operatorname{tr}\left(-r^{\mathfrak{a}}(f) \cdot{ }^{f} a_{i}{ }^{f} b_{i} \cdot r^{\mathfrak{a}}(f)^{-1}-a_{j} b_{j}-r^{\mathfrak{a}}(f) \cdot{ }^{f} a_{i}{ }^{f} b_{i} \cdot r^{\mathfrak{a}}(f)^{-1} \cdot a_{j} b_{j}\right) \\
& =-\operatorname{tr}\left(r^{\mathfrak{a}}(f) \cdot{ }^{f} a_{i}{ }^{f} b_{i} \cdot r^{\mathfrak{a}}(f)^{-1} \cdot a_{j} b_{j}\right) \\
& =-\operatorname{tr}\left(r^{\mathfrak{a}}(f) \cdot{ }^{f} a_{i}{ }^{f} b_{i} \cdot{ }^{f} J_{1}{ }^{-1} \cdot \overline{{ }^{t} r^{\mathfrak{a}}(f)} \cdot J_{1} \cdot a_{j} b_{j}\right) \quad \text { by (4.2) } \\
& =-\operatorname{tr}\left(r^{\mathfrak{a}}(f) \cdot{ }^{f} a_{i} \overline{{ }^{t f} a_{i}} \cdot \overline{{ }^{t} r^{\mathfrak{a}}(f)} \cdot J_{1} \cdot a_{j} \overline{{ }^{t} a_{j}} \cdot J_{1}\right) \\
& =-\overline{{ }^{t f} a_{i}} \cdot \overline{{ } r^{\mathfrak{a}}(f)} \cdot J_{1} \cdot a_{j} \cdot \operatorname{tr}\left(r^{\mathfrak{a}}(f) \cdot{ }^{f} a_{i} \overline{{ }^{t} a_{j}} \cdot J_{1}\right) \\
& =-{ }^{t} \overrightarrow{c_{1}} J_{1} \overline{\overrightarrow{c_{2}}} \cdot \overline{{ }^{t} a_{j}} J_{1} r^{\mathfrak{a}}(f) \cdot{ }^{f} a_{i} \\
& =-{ }^{t} \overrightarrow{c_{1}} J_{1} \overline{\overrightarrow{c_{2}}} \cdot{ }^{t} \overrightarrow{c_{2}} \cdot J_{1} \overline{\overrightarrow{c_{1}}} \\
& =\left\langle\left\langle c_{2}, c_{1}\right\rangle\right\rangle=\left\langle\left\langle c_{1}, c_{2}\right\rangle\right\rangle .
\end{aligned}
$$

Next, we consider the general case $\varphi_{1} \varphi_{2}=g f \psi_{i} f^{-1} \psi_{j} g^{-1}$ for $g \in \mathcal{M}_{g, 1}$. The pairing $\langle\langle\cdot, \cdot\rangle\rangle$ is $\mathcal{M}_{g, 1}$-equivariant by [S4, Lemma 4.3]; that is,

$$
\left\langle\left\langle g\left(c_{1}\right), g\left(c_{2}\right)\right\rangle\right\rangle=g\left(\left\langle\left\langle c_{1}, c_{2}\right\rangle\right\rangle\right) .
$$

Moreover, we see from [S3, Proposition 3.2] that

$$
\operatorname{tr}\left(r_{1}\left(g \varphi_{1} \varphi_{2} g^{-1}\right)\right)=g\left(\operatorname{tr}\left(r_{1}\left(\varphi_{1} \varphi_{2}\right)\right)\right) .
$$

This means that $\operatorname{tr}\left(r_{1}(\cdot)\right)$ is also $\mathcal{M}_{g, 1}$-equivariant. Therefore this completes the proof.

The Dehn twist along a bounding simple closed curve is called a BSCC map. From our previous paper [S3], it is known that any BSCC map $\varphi$ does not lie in the kernel of $r_{1}$, and the characteristic polynomial of the Magnus matrix of $\varphi$ is trivial:

$$
\operatorname{det}\left(\lambda I_{2 g}-r_{1}(\varphi)\right)=(\lambda-1)^{2 g}
$$

It follows that $\mathcal{K}_{g, 1}$ is not contained in the kernel of $r_{1}$, where $\mathcal{K}_{g, 1}$ denotes the subgroup generated by the BSCC maps. We remark that the characteristic polynomial of the Magnus matrix on $\mathcal{K}_{g, 1}$ is not always trivial (see [S4 for details).

Theorem 4.3 gives a characterization of the kernel of $r_{1}$ for the commutator of two BSCC maps.

Corollary 4.4. The commutator of two BSCC maps $\varphi_{1}, \varphi_{2}$ belongs to the kernel of $r_{1}$ if and only if the characteristic polynomial of the Magnus matrix of the product $\varphi_{1} \varphi_{2}$ is trivial. Here the Magnus matrix means the image of $r_{1}$ for a mapping class.

Proof. In general, if the characteristic polynomials of two matrices $A, B$ are trivial and $A$ commutes with $B$, then the characteristic polynomial of $A B$ is also trivial.

Suppose that the commutator of two BSCC maps $\varphi_{1}, \varphi_{2}$ belongs to the kernel of $r_{1}$, that is, $r_{1}\left(\varphi_{1}\right)$ commutes with $r_{1}\left(\varphi_{2}\right)$. Because the characteristic polynomial of the Magnus matrix for any BSCC map is trivial, we get

$$
\operatorname{det}\left(\lambda I_{2 g}-r_{1}\left(\varphi_{1} \varphi_{2}\right)\right)=(\lambda-1)^{2 g} .
$$

Conversely, suppose that the characteristic polynomial is trivial. Then we have

$$
-\operatorname{tr}\left(r_{1}\left(\varphi_{1} \varphi_{2}\right)\right)=-2 g \text {. }
$$


By Theorem 4.3, we conclude that $\left\langle\left\langle c_{1}, c_{2}\right\rangle\right\rangle=0$. This means that $\left\langle c_{1}, c_{2}\right\rangle_{H}=0$ or $\left\langle c_{2}, c_{1}\right\rangle_{H}=0$, because $\mathbb{Z}[H]$ is an integral domain. By virtue of Proposition 3.2 $\left\langle c_{1}, c_{2}\right\rangle_{H}=0$ gives $\left[\varphi_{1}, \varphi_{2}\right] \in \operatorname{ker} r_{1}$ and $\left\langle c_{2}, c_{1}\right\rangle_{H}=0$ gives $\left[\varphi_{2}, \varphi_{1}\right] \in \operatorname{ker} r_{1}$. This completes the proof.

\section{ACKNOWLEDGEMENTS}

The author would like to express his gratitude to Prof. Shigeyuki Morita for helpful suggestions and encouragement. He also would like to thank Prof. Yasushi Kasahara and Prof. Takayuki Morifuji for valuable discussions and advice.

\section{REFERENCES}

[Big] S. Bigelow, The Burau representation is not faithful for $n=5$, Geom. Topol. Vol.3 (1999) 397-404 MF $1725480(2001 \mathrm{j}: 20055)$

[B-B] S. Bigelow and R. Budney, The mapping class group of a genus two surface is linear, Alg. Geom. Topol. Vol.1 (2001) 699-708 MR1875613 (2002k:20065)

[Bir] J. Birman, Braids, Links and Mapping Class Groups, Annals of Math. Studies No. 82, Princeton University Press, 1975 MR 0375281 (51:11477)

[F] R. H. Fox, Free Differential Calculus I, Annals of Math. 57 (1953), 547-560 MR0053938 $(14: 843 \mathrm{~d})$

$[\mathrm{J}] \quad$ D. Johnson, A survey of the Torelli group, Contemporary Math. 20 (1983) 165-179 MR0718141 (85d:57009)

[K] M. Korkmaz, On the linearity of certain mapping class groups, Turkish J. Math. 24 (2000) 367-371 MF 1803819 (2001j:57020)

[M1] S. Morita, Abelian quotients of subgroups of the mapping class group of surfaces, Duke Math. J. 70 (1993), 699-726 MR.1224104(94d:57003)

[M2] S. Morita, Structure of the mapping class groups of surfaces: a survey and a prospect, Geom. Topol. Monogr. Vol.2: Proceedings of the Kirbyfest (1999) 349-406 MR1734418 (2000j:57039)

[S1] M. Suzuki, The Magnus representation of the Torelli group $\mathcal{I}_{g, 1}$ is not faithful for $g \geq 2$, Proc. Amer. Math. Soc. 130 (2002) 909-914 MR1866048 (2003c:57001)

[S2] M. Suzuki, Irreducible decomposition of the Magnus representation of the Torelli group, Bull. Austral. Math. Soc. 67 (2003) 1-14 MR1962955|(2004f:57001)

[S3] M. Suzuki, A class function on the Torelli group, Kodai Math. J. 26 (2003) 304-316 MR2018724

[S4] M. Suzuki, A geometric interpretation of the Magnus representation of the mapping class group, preprint

Graduate School of Mathematical Sciences, University of Tokyo, 3-8-1 Komaba, Meguro, TOKYO 153-8914, JAPAN

E-mail address: macky@ms.u-tokyo.ac.jp 